\title{
Analysis of spectral power distributions of fiuorescent lamps for the maximum luminous efficacy under given color and color rendering properties
}

\author{
Shoichiro YAMAGUCHI, Seishi SEKINE and Fujio KOBAYASHI \\ Numerical calculations were performed with an iterative linear program- \\ ming method for the purposes of predicting the optimum spectral power \\ distributions and the lighting characteristics of fluorescent lamps. Linear \\ programming was used in order to minimize the range containing local \\ solutions and insure convergence to the optimum solution. The optimum \\ spectral power distributions of fluoresent lamps making all of special color \\ rendering indices of $R_{1}$ to $R_{8} 95$ or more must be composed of four narrow \\ spectral bands heving a radiation peak af $470,530,580$ and $620 \mathrm{~nm}$.
}

\section{Introduction}

The luminous efficacy, chromaticity and color rendering properties of fluorescent lamps depend on the SPD's (spectral power distributions) of the lamps. It is the purpose for the developments of the lamps and the fluorescent lamps especially to make luminous efficacy maximum under a given color and CRI (color rendering index). Luminous efficacy of lamps is the product of these radiant efficiency by luminous efficacy of the radiation. The radiant efficacy can be improvement by developments in physical and chemical properties of various materials. The luminous efficacy can be enhanced by actualizing the ideal SPD's through selection of adequate luminous materials as regards fluorescent lamps. Analysis of the optimum SPD's which give the maximum luminous efficacy under the prescribed constraints of chromaticity and color rendering properties can be made in the following two ways with the aid of digital computers:

(1) A method of analysis with constraints put on the peak wavelength, wavelength band width and the like of the luminous components to be combined in consideration of the characteristics of luminous materials.

(2) A method of analysis with constraints put on the radiant flux, chromaticity and color rendering properties, but not at all on the shape of SPD.

(Until now, the method (1) has been used to predict the optimum SPD of narrow-band type fluorescent lamps, and the three-band type lamp has been developed based on it. ${ }^{12)}$ ).

Problems to which the method (2) is applicable are, mathematically conditional extremum problems and can be dealt with effectively by mathematical programming.

In cases where the optimum SPD's are sought which maximize the objective function or the total luminous flux under the constraints of the radiant flux, chromaticity and CRI (color rendering index)

* A part of this paper was described in J. Illum. Engn. Inst. Jun. 67, 6(1983) of fluorescent lamps, the constraint equations pertaining to the chromaticity and CRI are nonlinear and nonlinear programming (NLP) is generally applicable here. Water ${ }^{3)}$ and Fuchida et al.4) reported on NLP analysis of the optimum SPD's of fluorescent lamps.

Walter set up the objective function and constraint equations for 4 kinds of fluorescent lamps specifide by ANSI C78.376-1969, WW, W, CW and $\mathrm{D},{ }^{5)}$ and analyzed the optimum SPD's. He regarded four monochromatic components at $460,530,580$ and $620 \mathrm{~nm}$ of wavelength, as fundamental to the SPD's which maximize the total luminous flux under the constraints $R_{a} \geqq 90$ or $R_{a} \geqq 95$. However, spectral powers other than those four monochromatic components appear in the SPD's he obtained and it has not been confirmed whether or not these four monochromatic components alone can actually satisfy $R_{a} \geqq 90$ or $R_{a} \geqq 95$. Walter pointed out that the supersurface containing the optimum solution is fairly flat and it is possible to make small changes in the SPD's without greatly affecting the luminous efficacy or the CRI.

Fuchida et al. predicted the complications that the optimum solution may vary with the algorithm applied in the problems such as above which may yield a large number of local solutions. Therefore, they obtained the optimum solution by applying five kinds of NLP algorithms to the objective function and constraints approximately equal to those set up by Walter. They reported that analysis by each algorithm converged into almost the same optimum solution and a spectral power appeared at $490 \mathrm{~nm}$ where none had been reported by Walter.

We have studied the differences between the two works cited above and analyzed the optimum SPD's in the whole range of chromaticity specified in JIS Z9112-1976 ${ }^{\text {) }}$ in order to clarify in more detail the characteristics of the optimum SPD's such as monochromatic components, luminous flux, chromaticity and CRI.

In carrying out the analysis, we consider it is better to constrain the chromaticity not by chromaticity range but by chromaticity coordinates 
in order to minimize the range containing local solutions and insure convergence to the optimum solution. If this is done, the constraints on the chromaticity become linear. On the other hand, the number of sample points increases markedly. It is therefore necessary to shorten the time required for calculation of the optimum SPD's for each sample point (chromaticity coordinates). To this end, the nonlinear constraint equations relating to the color rendering index were modified to forms to which LP (linear programming) is applicable and LP was applied repeatedly to realize convergence to the optimum solution. LP does not require calculation of gradient vectors which is essential for NLP and it is expected that LP will complete the calculation in a shorter time than NLP where LP needs to be repeated only a limited number of times.

In this paper, we report on the algorithm we used for optimization and the characteristics of the optimum SPD's we found on 40-watt fluorescent lamps.

\section{Objective function and con- straint equations}

(1) Objective function maximizing the total luminous flux

The visible wavelength range $400 \sim 700 \mathrm{~nm}$, was divided into 31 monochromatic components, each $10 \mathrm{~nm}$ in wavelength band width.

$$
\begin{aligned}
& \phi=K_{m} \Delta \lambda \sum_{j=1}^{31} V_{j} P_{j}+\varphi_{H g} \\
& \varphi_{H g}=K_{m} \sum_{i=1}^{4} V_{i} c_{i}\left(r_{1}+r_{2}\right)
\end{aligned}
$$

(The proportion of the mercury line spectrum was assumed constant and this equation was adopted for convenience' sake.)

where: $\phi=$ total luminous flux in $1 \mathrm{~m} ; K_{m}=$ maximum luminous efficacy in $1 \mathrm{~m} / \mathrm{W} ; \Delta \lambda=$ wavelength band width or $10 \mathrm{~nm} ; V_{j}, V_{i}=$ standard spectral luminous efficiency; $P_{j}=$ spectral power of fluorescent material in $W ; \varphi_{H g}=$ total luminous flux caused by the mercury line spectrum in $\mathrm{lm} ; r_{1}, r_{2}=$ the ultraviolet exciting power in 185 and $254 \mathrm{~nm}$ in $W ; c_{i}=$ relative powers of visible spectral line at $405,436,546$ and $578 \mathrm{~nm}$ in the ratio to the sum of $r_{1}$ and $r_{2}$.

Here, values of $0.0090,0.0212,0.0112$ and 0.0032 were adopted for $c_{1}, c_{2}, c_{3}$ and $c_{4}$ respectively, while values of 3.49 and $21.3 \mathrm{~W}^{4)}$ for $r_{1}$ and $r_{2}$ respectively.

(2) Constraint equation for radiant flux

The constraint on radiant flux from the excited fluorescent materials is expressed as

$$
\Delta \lambda \sum_{j=1}^{31} \lambda_{j} P_{j}=\sum_{k=1}^{2} \lambda_{k} r_{k} q_{k}
$$

where: $\lambda_{j}=$ wavelength of fluorescence in $\mathrm{nm} ; \lambda_{k}=$ wavelength of ultraviolet excitation in $\mathrm{nm} ; q_{k}=$ quantum efficiency of fluorescent material.

Here, $q_{1}(185 \mathrm{~nm})=q_{2}(254 \mathrm{~nm})=0.77$ was adopted for $q_{k}^{4)}$.

\section{(3) Constraint equation for chroma- ticity}

The constraints on the chromaticity $\left(x_{k}, y_{k}\right)$ are expressed as

$$
\left.\begin{array}{c}
x_{k}=\frac{\Delta \lambda \sum_{j=1}^{31} P_{j} \bar{x}_{j}+\sum_{i=1}^{4} c_{i}\left(r_{1}+r_{2}\right) \bar{x}_{i}}{\Delta \lambda \sum_{j=1}^{31} P_{j} \bar{s}_{j}+\sum_{i=1}^{4} c_{i}\left(r_{1}+r_{2}\right) \bar{s}_{i}} \\
y_{k}=\frac{\Delta \lambda \sum_{j=1}^{31} P_{j} \bar{y}_{j}+\sum_{i=1}^{4} c_{i}\left(r_{1}+r_{2}\right) \bar{y}_{i}}{\Delta \lambda \sum_{j=1}^{31} P_{j} \bar{s}_{j}+\sum_{i=1}^{4} c_{i}\left(r_{1}+r_{2}\right) \bar{s}_{i}}
\end{array}\right\}
$$

where: $\bar{x}_{j}, \bar{y}_{j}, \bar{z}_{j}, \bar{x}_{i}, \bar{y}_{i}$ and $\bar{z}_{i}$ are tristimulus values.

\section{(4) Constraint equation for CRI}

The constraints on the special CRI's are expressed as

$$
R_{i} \geqq R_{c} \quad(i=1-8)
$$

where: $R_{i}=$ special CRI of a test color $(i)$ for calculation of the general CRI; $R_{e}=$ constant.

The constraint on the general CRI $R_{a}$, as given in equation (5) below, was fundamentally not adopted here in order to keep a given value of $R_{i}$ from becoming extraordinary small relative to value of $R_{a}$ assumes large values and to minimize fluctuations in $R_{i}$.

$$
R_{a}=\frac{1}{8} \sum_{i=1}^{8} R_{i} \geqq R_{c} \quad(i=1-8)
$$

However, calculations were carried out for several examples by equation (5) and the results were compared with those obtained from equation (4). No constraint was put on the special CRI's $R_{i}$ ( $i=9-$ 15). In the discussions which follow, the subscript $i$ in $R_{i}$ is $1-8$ unless otherwise stated.

As to the constraints, we differ from Walter mainly in those restricting the chromaticity and CRI as follows. We fixed chromaticity to a selected point and calculated the CRI by the method described in CIE (1st edition) JIS. On the other hand, Walter limited the chromaticity to the MacAdam ellipses specified by $\mathrm{ANSI}^{5)}$ and calculated the CRI by the method in CIE (2nd edition), We used 0.77 as quantum efficiency of the fluorescent material whereas Walter used 1. Also, our total luminous flux of the mercury spectral lines is somewhat different from his.

\section{Algorithm for optimization by repeated applications of LP}

The values of $R_{i}$ are obtained from the following equations. According to CIE (1st edition), one calculates $R_{i}$ with incorporation of the lightness index. The equation (6) is used here since it gives approximately the same results as the method of CIE (2nd edition). 


$$
\begin{aligned}
\Delta U_{i} & =\left(u_{k i}-u_{k}\right)-\left(u_{o i}-u_{i}\right) \\
\Delta V_{i} & =\left(v_{k i}-v_{k}\right)-\left(v_{o i}-v_{k}\right) \\
\Delta E_{i} & =800 \sqrt{\Delta U_{i}^{2}+\Delta V_{i}^{2}} \\
R_{i} & =100-4.6 \Delta E_{i}
\end{aligned}
$$

where: $u_{k i}, v_{l k i}=\mathrm{UCS}$ chromaticity coordinates of test color $(i)$ under sample light source,

$u_{o i}, v_{o i}=\mathrm{UCS}$ chromaticity coordinates of test color (i) under reference illuminant,

$u_{k}, v_{k}=\mathrm{UCS}$ chromaticity coordinates of sample light source, and

$u_{o}, v_{o}=\mathrm{UCS}$ chromaticity coordinates of reference illuminant.

The constraint equation for $\Delta E_{i}$ is obtained from equation (4) as follows:

$$
\Delta E_{i} \geqq \Delta E_{c} \frac{100-R_{c}}{4.6}
$$

\section{Procedure 1}

LP is used to calculate the SPD's which satisfy given chromaticity coordinates $\left(u_{k}, v_{k}\right)$ under the following constraints and maximize the total luminous flux:

$$
\left|\Delta U_{i}\right| \leqq \Delta E_{e}, \quad\left|\Delta V_{i}\right| \leqq \Delta E_{e}
$$

In general, an increase in total luminous flux causes a decrease in CRI. Therefore, $\Delta U_{i}, \Delta V_{i}$ and $\triangle E_{i}$ are calculated for the SPD's obtained and designated as $\Delta U^{\prime}, \Delta V^{\prime}{ }_{i}$ and $\Delta E^{\prime}{ }_{i}$ respectively. Then, because of the constraints of equation (8) at least one or more of $\Delta U^{\prime}{ }_{i}$ and $\Delta V^{\prime}{ }_{i}$ become equal to $\Delta E^{\prime}{ }_{i}$ and:

$$
\Delta E_{c}<\Delta E_{m}^{\prime}<\sqrt{2} \Delta E_{c}
$$

where $\Delta E^{\prime}{ }_{m}$ is the maximum value of $\Delta E^{\prime}{ }_{i}$. Thus, the constraint equation (7) is not satisfied.

\section{Procedure}

LP is used to calculate the SPD's which satisfy given chromaticity coordinates $\left(u_{k}, v_{k}\right)$ under the following constraints and maximize the total luminous flux:

$$
\left.\begin{array}{l}
\text { If }\left|\Delta U_{i}^{\prime}\right| \geqq\left|\Delta V_{i}^{\prime}\right|, \\
\text { then }\left|\Delta U_{i}\right|=\left|\Delta V_{i}\right| \leqq\left|\Delta U_{i}^{\prime}\right| \frac{\Delta E_{c}}{\Delta E_{i}{ }^{\prime}} \\
\text { If }\left|\Delta U_{i}^{\prime}\right|<\left|\Delta V_{i}^{\prime}\right|, \\
\quad \text { then }\left|\Delta U_{i}\right|=\left|\Delta V_{i}\right| \leqq\left|\Delta V_{i}^{\prime}\right| \frac{\Delta E_{c}}{\Delta E_{i}{ }^{\prime}}
\end{array}\right\}
$$

The values of $\Delta U_{i}, \Delta V_{i}$ and $\Delta E_{i}$ are calculated for the SPD's obtained and designated as $\Delta U^{\prime \prime}$, $\Delta V^{\prime \prime}{ }_{i}$ and $\Delta E^{\prime \prime}{ }_{i}$ respectively.

Then, the following equations hold:

$$
\left.\begin{array}{l}
\text { If }\left|\Delta U_{i}{ }^{\prime}\right| \geqq\left|\Delta V_{i}{ }^{\prime}\right|, \\
\quad \text { then } \Delta E_{i}^{\prime \prime} \leqq 800 \sqrt{\left(\Delta U_{i}^{\prime} \frac{\Delta E_{c}}{\Delta E_{i}}\right)^{2}+\left(\Delta U_{i}^{\prime} \frac{\Delta E_{c}}{\Delta E_{i}{ }^{\prime}}\right)^{2}} \\
\text { If }\left|\Delta U_{i}^{\prime}\right|<\left|\Delta V_{i}{ }^{\prime}\right|, \\
\quad \text { then } \Delta E_{i}^{\prime \prime} \leqq 800 \sqrt{\left(\Delta V_{i}^{\prime} \frac{\Delta E_{e}}{\Delta E_{i}}\right)^{2}+\left(\Delta V_{i}^{\prime} \frac{\Delta E_{c}}{\Delta E_{i}^{\prime}}\right)^{2}}
\end{array}\right\}
$$

Furthermore, if $\Delta E^{\prime}{ }_{i} \simeq E_{c}$, the following equations hold true as $\Delta U^{\prime}{ }_{i}$ is generally proportional to $\Delta V^{\prime}{ }_{i}$ :

$$
\begin{aligned}
& \text { If }\left|\Delta U_{i}^{\prime}\right| \geqq\left|\Delta V_{i}^{\prime}\right| \text {, } \\
& \text { then } \begin{aligned}
\Delta E_{i}^{\prime \prime} & \leqq 800 \sqrt{\left(\Delta U_{i}^{\prime} \frac{\overline{\Delta E_{c}}}{\Delta E_{i}^{\prime}}\right)^{2}+\left(\Delta V_{i}^{\prime} \frac{\Delta E_{c}}{\Delta E_{i}^{\prime}}\right)^{2}} \\
& \leqq 800 \sqrt{\left(\Delta U_{i}^{\prime} \frac{\Delta E_{c}}{\Delta E_{i}}\right)^{2}+\left(\Delta U_{i}^{\prime} \frac{\Delta E_{c}}{\Delta E_{i}^{\prime}}\right)^{2}}
\end{aligned} \\
& \text { If }\left|\Delta U_{i}^{\prime}\right|<\left|\Delta V_{i}^{\prime}\right| \text {, } \\
& \text { then } \Delta E_{i}^{\prime \prime} \leqq 800 \sqrt{\left(\Delta U_{i}^{\prime} \frac{\Delta E_{c}}{\Delta E_{i}^{\prime}}\right)^{2}+\left(\Delta V^{\prime} \frac{\Delta E_{c}}{\Delta E_{i}}\right)^{2}} \\
& \leqq 800 \sqrt{\left(\Delta V^{\prime} \frac{\Delta E_{c}}{\Delta E_{i}^{\prime}}\right)^{2}+\left(\Delta V^{\prime} \frac{\Delta E_{c}}{\Delta E_{i}}\right)^{2}}
\end{aligned}
$$

where:

$$
800 \sqrt{\left(\Delta U_{i}^{\prime} \frac{\Delta E_{c}}{\Delta E_{i}}\right)^{2}+\left(\Delta V_{i}^{\prime} \frac{\Delta E_{c}}{\Delta E_{i}}\right)^{2}}=\Delta E_{c}
$$

If $\Delta E^{\prime}{ }_{m}$, the maximum values of $\Delta E^{\prime}$, becomes so nearly equal to $\Delta E_{c}$ as to make equation (12) hold true after execution of Procedure 1 , the following relation will be satisfied after execution of Procedure 2:

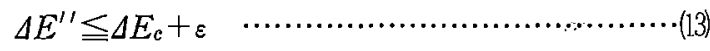

where $\epsilon$ is a sufficiently small positive number. Since an increase in total luminous flux generally causes a decrease in CRI, at least one or more of $\Delta E^{\prime \prime}{ }_{i}$ can be expressed as follows:

$$
\begin{aligned}
& \Delta E_{i}{ }^{\prime \prime}=\Delta E_{c}+\varepsilon \ldots \ldots \ldots \ldots \ldots \ldots \ldots \ldots \ldots \ldots \ldots \ldots \ldots \ldots \ldots \ldots \ldots \ldots \ldots \\
& \text { Therefore, the SPD's then satisfy the constraint }
\end{aligned}
$$
of equation (7) and maximize the total luminous flux.

If equation (13) is not satisfied by execution of Procedure 1 and 2, a convergence can be obtained by repeating further only Procedure 2. Our experiences indicate that Procedure 2 needs to be repeated from 2 to 10 times when $\varepsilon \simeq 10^{-4} \Delta E_{c}$ produced a convergence.

\section{Optimization of SPD's}

Optimization was first done on the SPD's of 31 monochromatic components, each $10 \mathrm{~nm}$ in wavelength band width, of the visible wavelength range $400 \sim 700 \mathrm{~nm}$. The study was restricted to the four chromaticity range, WW $30, \mathrm{WW}, \mathrm{W}$ and $\mathrm{D}$, specified in JIS Z9112-1976. $\left.{ }^{\circ}\right)$

\section{1 Chromaticity range and total Iuminous flux}

Figure 1 showes the chromaticity range in which the SPD's satisfying $R_{i} \geqq 90$ or $R_{i} \geqq 95$ may exist. This chromaticity range extends from the Planckian locus $P$ at center approximately symmetrically toward the green side $(+)$ and the violet side $(-)$ along each isotemperature line. The width of this range increases as the CCT (correlated color temperature) rises. It is only natural that, as the constraints on the CRI become severe, the chromaticity range containing SPD's satisfying such severe constraints becomes narrower. The locus $\mathrm{L}$ in Fig. 1. represents the chromaticity locus of the SPD's which maximize the total luminous flux at each $\mathrm{CCT}$ in the chromaticity range satisfying 


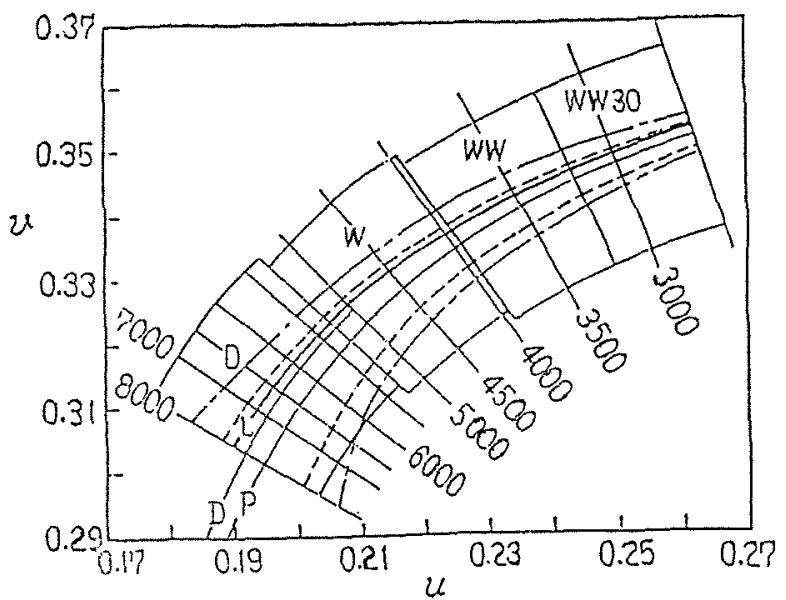

Fig. 1 The chromatisity ranges where the spectral power distributions satisfying $R_{i} \geqq 90$ or $R_{i} \geqq 95$ exist (enclosed by dot-and-dash lines or broken lines) and the chromaticity locus $L$ maximizing the total luminous flux under $R_{i} \geqq 95 \quad(i=1-8)$ at given correlated color temperature.

$R_{i} \geqq 95$. The locus $\mathrm{L}$ runs near the green side boundary of the range.

Figure 2 shows the maximum total luminous flux under the constraint $R_{i} \geqq 95$ as a function of CCT. The discontinuity in CCT between 5300 and $5400 \mathrm{~K}$ is due to the calculating procedure in which a Planckian radiator is used the reference illuminant at $5300 \mathrm{~K}$ and below whereas CIE daylight is used at $5400 \mathrm{~K}$ and above. This discontinuity will appear in some of the later figures for the same reason. The total luminous flux is large in a low CCT range, for example $W W 30$ and $W W$, and diminishes as the CCT rises from $W$ to $D$. The maximum of about $3480 \mathrm{~lm}$ appears in the CCT range of 3300 to $3500 \mathrm{~K}$ and the luminous efficacy there is about $87 \mathrm{~lm} / \mathrm{W}$.

Figure 3 shows the relation between the maximum total luminous flux and the chromaticity deviation from the Planckian locus under the constraint of $R_{i} \geqq 95$ at CCT of 3500,4500 and $6500 \mathrm{~K}$. The total luminous flux increases as the chromaticity moves on a given isotemperature line from the violet side boundary to the green side boundary and reaches a maximum immediately before the green side boundary. Also, the total luminous flux decreases sharply as the chromaticity comes very close to either of the boundaries.

\subsection{Optimum SPD's}

Figure 4 shows each spectral power of the SPD's which maximize the total luminous flux under the constraint of $R_{i} \geqq 95$ at given CCT's. This figure reveals the following features of the optimum SPD's satisfying $R_{i} \geqq 95$ in the whole range of chromaticity specified in JIS Z9112-1978.6)

(1) None of the optimum SPD needs radiation outside the wavelength range of $460-620 \mathrm{~nm}$ (ex-

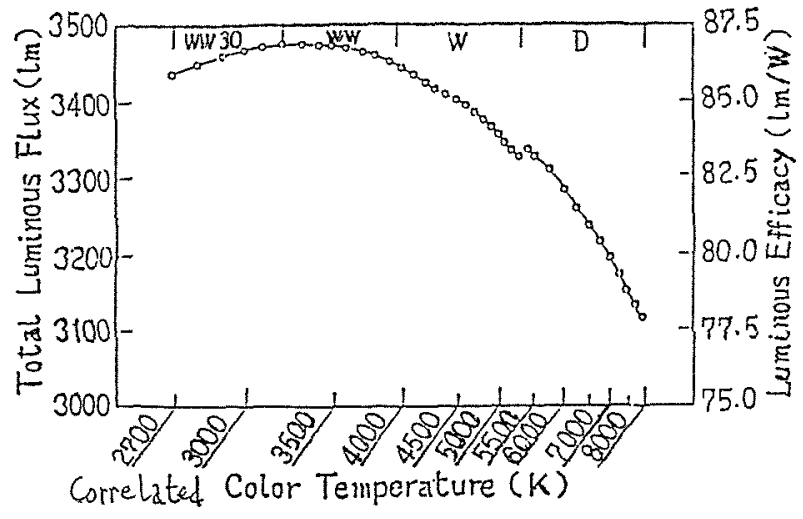

Fig. 2 The maximum total luminous flux under $R_{i} \geqq 95(i=1-8)$ as a function of correlated color temperature.

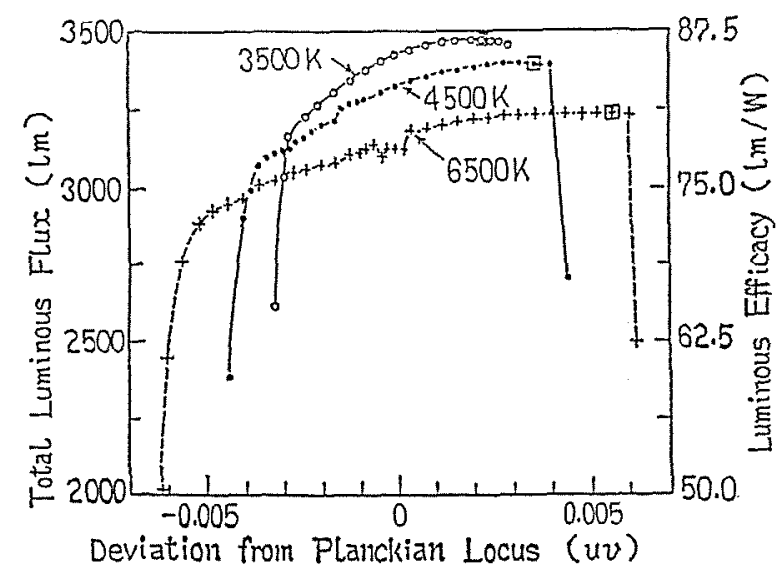

Fig. 3 Relation between total luminous flux and deviation from the Planclian locus $R_{i} \geqq 59(i=1-8) ; \square$ designating maximum).

cluding the mercury spectral line).

(2) There are four monochromatic components of primary importance, $470,530,580$ and $620 \mathrm{~nm}$, and two of secondary importance, 460 and $520 \mathrm{~nm}$.

The first feature agrees with Walter's findings. As for the second feature, Walter concluded four monochromatic components of $460,530,580$ and $620 \mathrm{~nm}$ to be of primary importance from his calculations performed under the constraints $R_{i} \geqq 90$ and $R_{i} \geqq 95$. We also obtained the results like Walter under $R_{i} \geqq 90$ that the spectral power at $460 \mathrm{~nm}$ is greater than that at $470 \mathrm{~nm}$ in the whole CCT range.

In spite of the above-mentioned differences in the constraints between Walter and ours, both arrived at the optimum SPD's with almost the same features.

The ultraviolet excitation power, relative spectral distribution of the mercury line spectrum and the (quantum) efficiency of fluorescent materials considerably depend on the lamp construction and the performance of the component materials. A comparative study was therefore made on the optimum 


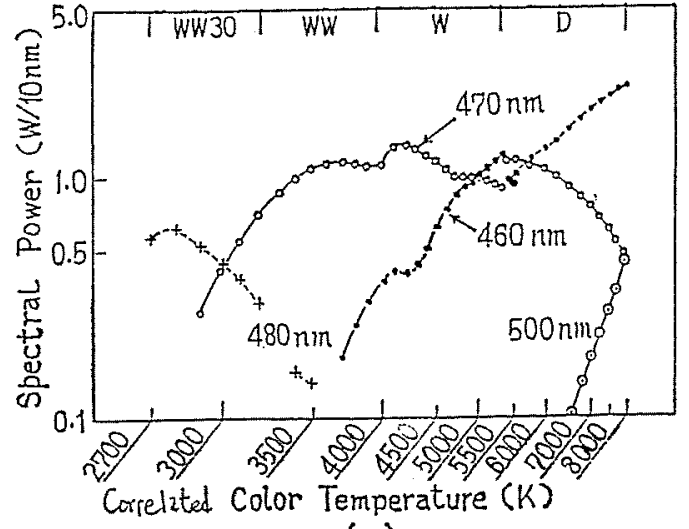

(a)

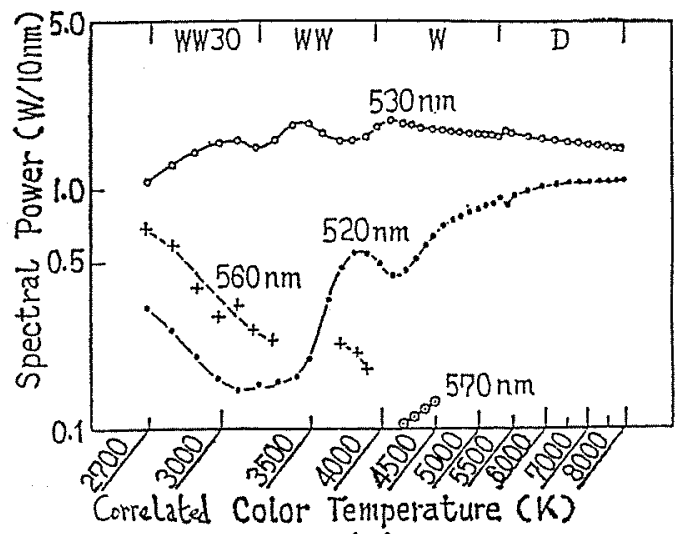

(b)

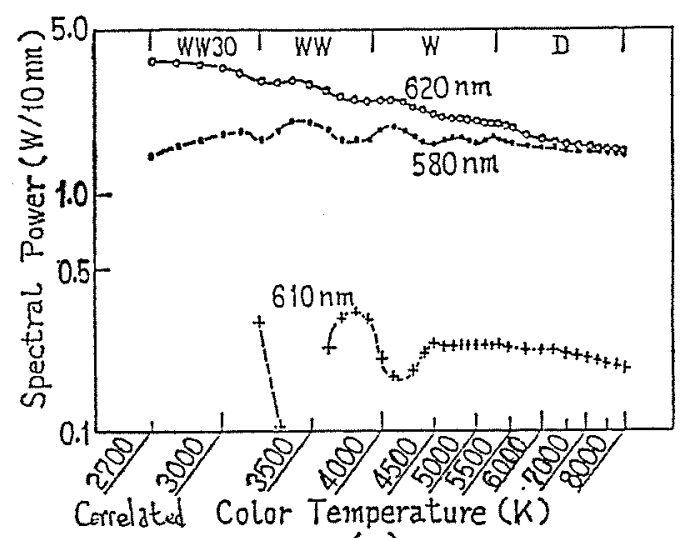

(c)

Fig. 4 The monochromatic components of spectral power distributions maximizing the total luminous flux under $R_{i} \geqq 59 \quad(i=1-8)$ at given correlated color temperatures (excluding the mercury spectral line).

SPD's for different values of above-mentioned parameters. The results indicate that, although the relative special distributions are different, there was no difference in monochromatic components at which the spectral powers appeared.

Inspection of Fig. 2 and 4 shows that the number of monochromatic components is six at the most (excluding the mercury spectral lines here and same as above) in the CCT range of $3300-3500 \mathrm{~K}$ where the total luminous flux assumes the maximum value or a value close to it whereas this number becomes greater in other CCT ranges. Also, Walter obtained the results that, under the constraints $R_{i} \geqq R_{c}$ or $R_{a} \geqq R_{c}$, the total luminous flux increases but the number of monochromatic components decreases as $R_{c}$ decreases from 95 to 90 down to 50 . These findings lead to the following conclusion: in conversion of ultraviolet-excited radiant powers of a fluorescent lamp into spectral powers of the visible wavelength range by means of a fluorescent material, the luminous efficacy can be enhanced and a given CRI's can be satisfied by minimizing the number of monochromatic components and concentrating the spectral powers to specific monochromatic components. An increase in the number of monochromatic components raises the CRI but lowers the luminous efficacy.

Figure 5(a) shows the SPD which maximizes the total luminous flux under $R_{i} \geqq 95$ at $4500 \mathrm{~K}$. The deviation from the Planckian locus is $0.0037 \mathrm{uv}$. Fig. 5(b) shows the SPD which maximizes the total luminous flux under $R_{i} \geqq 95$ at $4500 \mathrm{~K}$ and -0.0040 uv. Comparison of Fig. 5(a) and 5 (b) indicates that the total luminous flux in (a) is greater than that in (b) by $487 \mathrm{~lm}$ or by about $14 \%$ and that the SPD in (a) has 8 monochromatic components and is over the range of $460 \sim 620 \mathrm{~nm}$ whereas that in (b) has 9 monochromatic components and is over the range of $430 \sim 630 \mathrm{~nm}$. Furthermore, the proportion of spectral powers in the wavelength range of high spectral luminous efficiency to the total spectral powers is greater in (a) than in $(b)$.

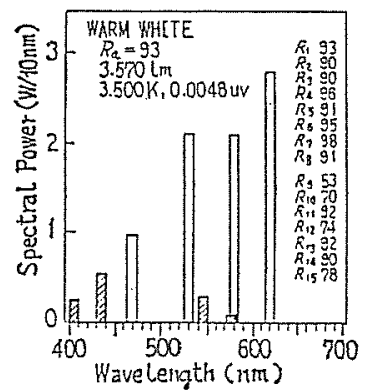

(a)

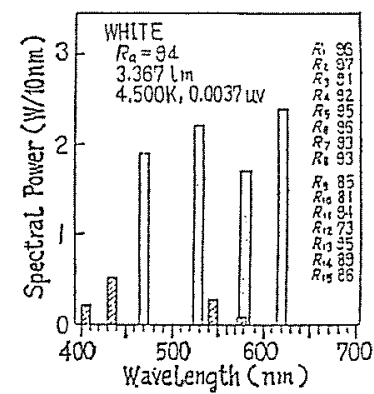

(b)
Fig. 5 Spectral power distributions satisfying $R_{i} \geqq 95 \quad(i=1-8)$ at $4500 \mathrm{~K}$ (hached bars for the medcury spectral lines).

Thus, of the SPD's obtained at a given CCT under the same constraints of the CRI, the SPD maximizing the total luminous flux has the smallest number of monochromatic components and a higher proportion of spectral powers falling the wavelength range of high spectral luminous efficiency. The chromaticity of the SPD maximizing the total luminous flux at a given $\mathrm{CCT}$ is present near the green side boundary and this is due to the fact that the monochromatic component of $555 \mathrm{~nm}$ at which the spectral luminous efficiency is maximum is 
yellowish green (see Fig. 3).

Figure 6, (a) and (b), shows typical SPD's maximizing the total luminous flux under $R_{i} \geqq 95$ at given CCT's. Fig. 6, (c) and (d), shows SPD's maximizing the total luminous flux under $R_{a} \geqq 95$ in the same chromaticity coordinates as those for SPD's in (a) and (b).

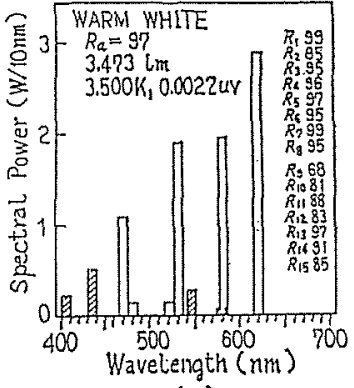

(a)

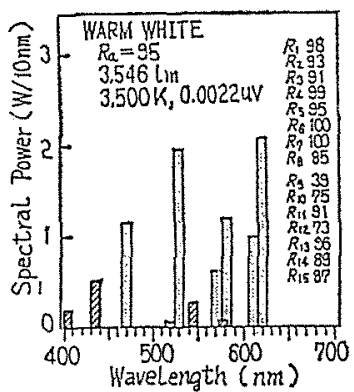

(c)

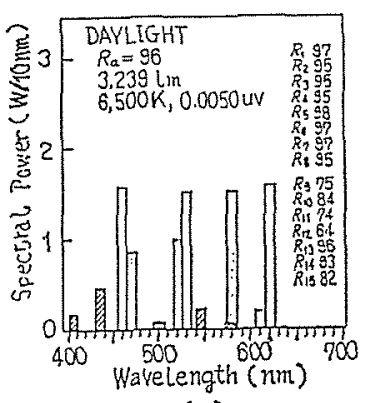

(b)

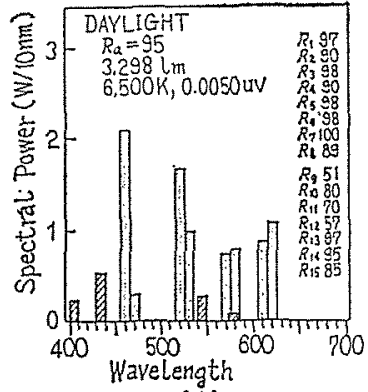

(d)
Fig. 6 Spectral power distributions under $R_{i} \geqq 95$ ( $i=1-8$ ) in (a) and (b) and those under $R_{a} \geqq 95$ in (c) and (d) (hached bars for mercury spectral lines).

The wavelength range for the SPD's under $R_{a} \geqq 95$ is $460 \sim 620 \mathrm{~nm}$ just as under $R_{i} \geqq 95$ and the number of monochromatic components is the same at 3500 and $6500 \mathrm{~K}$ respectively. There is a difference, however, that the spectral powers at 580 and $620 \mathrm{~nm}$ under $R_{a} \geqq 95$ are weaker than the corresponding ones under $R_{i} \geqq 95$ and strong spectral powers appear at 570 and $610 \mathrm{~nm}$ under $R_{a} \geqq 95$. On the other hand, comparison of the CRI shows that the special CRI's $R_{i}$ ( $\left.i=9-15\right)$ fluctuate less under $R_{i} \geqq 95$ than under $R_{a} \geqq 95$. As a consequence, a comprehensive assessment based on the SPD, total luminous flux and CRI is that the optimum SPD under $R_{i} \geqq 95$ is superior to that under $R_{a} \geqq 95$.

The spectral power of $490 \mathrm{~nm}$ pointed out by Fuchida et al. appeared weakly in the SPD at $2700 \mathrm{~K}$ of the SPD's maximizing the total luminous flux under $R_{i} \geqq 95$ at given CCT's, but not at any other CCT. However, a spectral power of $480 \mathrm{~nm}$ appeared in the SPD's having the chromaticity coordinates on the violet side of the Planckian locus in the chromaticity range satisfying $R_{i} \geqq 95$. This spectral power became stronger as the deviation from the Planckian locus to the violet side increased (see Fig. 5).

\subsection{Special CRI's}

The special CRI's $R_{i}$ ( $\left.i=9-15\right)$ fluctuated widely from one index to another because of no imposition of constraints.

Figure 7 shows the relationship between $R_{i}$ ( $i=$ 9-15) and CCT for the SPD's maximizing the total luminous flux under $R_{i} \geqq 95(i=1-8)$ at each $C C T$ (see Fig. 4).

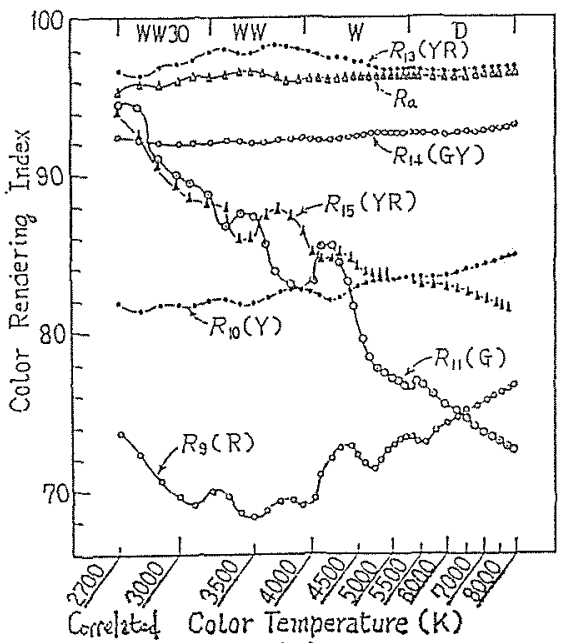

(a)

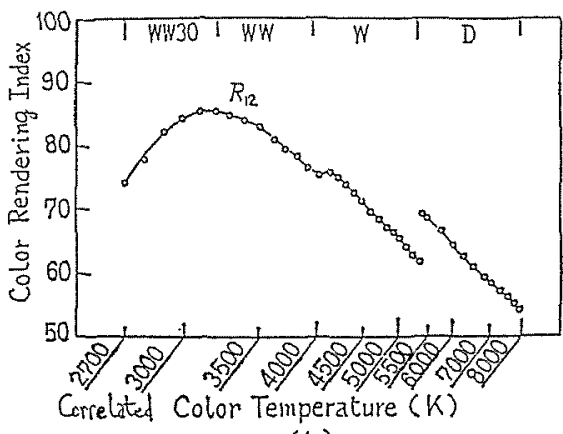

(b)

Fig. 7 Special color rendering indices $R_{i} \quad(i=9-15)$ of spectral power distributions maximizing the total luminous flux under $R_{i} \geqq 95(i=1-8)$ at given correlated color temperatures.

In the whole CCT range of $2700-8000 \mathrm{~K}, R_{13}$ (complexion) is greater than 95 and $R_{14}$ (foliage) and $R_{10}(Y)$ are relatively constant at $92-95$ and 82-83 respectively. The CRI's $R_{11}$ (G) $R_{15}$ (actual complexion) are the highest at $2700 \mathrm{~K}$, both 94 , and decrease as the CCT rises. The index $R_{1 z}$ (B) is 85 , the highest, near $3200 \mathrm{~K}$ and decreases markedly at higher CCT's. The index $R_{9}(\mathrm{R})$ is the lowest at 68 in the WW range and, outside this range, it increases as the CCT rises.

Inspection of Fig. 2 and 7 indicates that SPD's maximizing the total luminous flux under $R_{i} \geqq 95$ at each CCT shows a high luminous efficacy in the low CCT range corresponding to WW 30 and WW 
at the same time high values of special CRI's there as a whole.

\section{Optimization of SPD's of Spe- cific monochromatic compo- nents}

Optimization of SPD's of 31 monochromatic components obtained by dividing the visible wavelength range $400 \sim 700 \mathrm{~nm}$ by a wavelength band width of $10 \mathrm{~nm}$ showed that fundamental monochromatic components maximizing the total luminous flux under $R_{i} \geqq 95$ at each CCT are $470,530,580$ and $620 \mathrm{~nm}$. Optimization was performed over again on these four monochromatic components.

\subsection{Chromaticity range and total luminous flux}

Figure 8 shows the chromaticity range satisfying $R_{i} \geqq 90$. This range covers CCT's $2700-4600 \mathrm{~K}$, contains the Planckian locus $P$ and is slightly drawn toward the green side. The chromaticity locus $L$ of the SPD's maximizing the total luminous flux at each CCT is the green side boundary of this range.

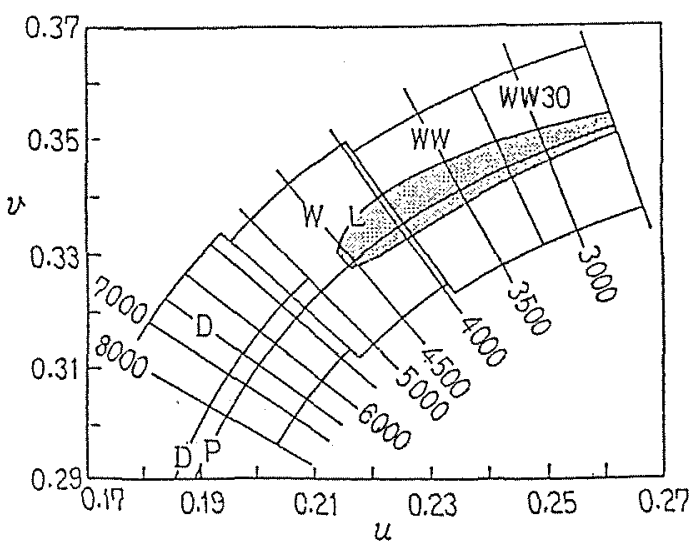

Fig. 8 The chromaticity range (shaded area) where four monochromatic components of 470,530 , 580 and $620 \mathrm{~nm}$ alone satisfy $R_{i} \geqq 90$ ( $i=1-8$ ) and the chromaticity locus $L$ giving the optimum spectral power distributions.

Here, there existed no chromaticity range where the above-mentioned four monochromatic components alone satisfy $R_{i} \geqq 95$. Addition of the monochromatic component of $460 \mathrm{~nm}$ to these four, namely existence of at least five monochromatic components, is necessary to satisfy $R_{i} \geqq 95$. In order to anticipate a still wider chromaticity range, six monochromatic components by further addition of the monochromatic component of $520 \mathrm{~nm}$ are necessary. On the other hand, a minimum of four monochromatic components is required to satisfy $R_{i} \geqq 90$ and a combination of the first mentioned four monochromatic components alone can satisfy this constraint.
It was impossible to find a combination of other four monochromatic components to satisfy $R_{i} \geqq 90$ than the foregoing.

Figure 9 shows the maximum total luminous flux as a function of CCT. The total luminous flux is roughly constant at $3565 \pm 15 \mathrm{~lm}$ in the low temperature ranges of WW 30 and WW and diminishes sharply as the CCT rises in the $W$ range.

\subsection{SPD and CR}

Figure 10 shows each spectral power of the SPD's which maximizing the total luminous flux under $R_{i} \geqq 90$ at given CCT.

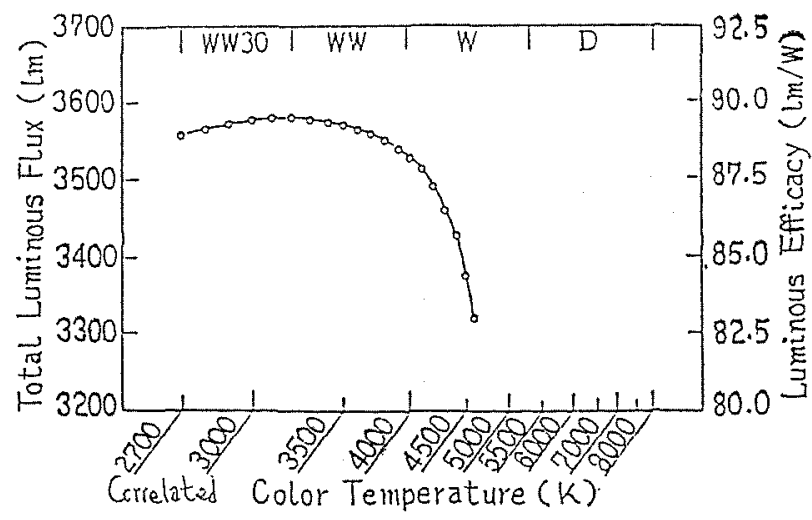

Fig. 9 The maximum total luminous fux of spectral power distributions of 470,530 , 580 and $620 \mathrm{~nm}$ alone satisfying $R_{i} \geqq 90$ $(i=i-8)$ as a function of correlated color temperature.

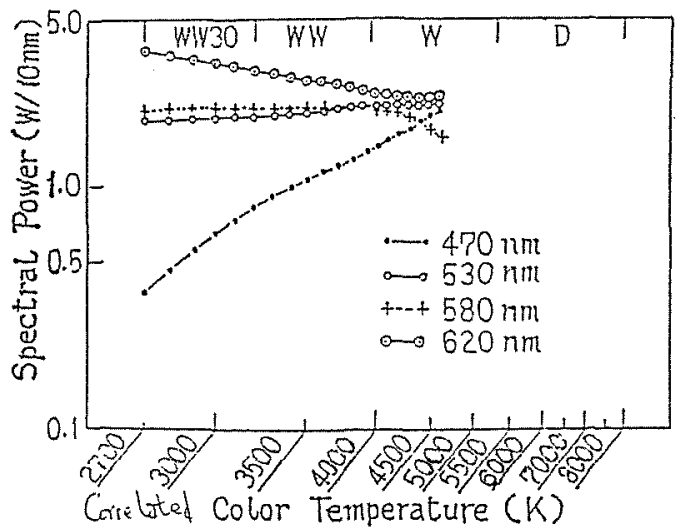

Fig. 10 Each spectral power of the spectral power distributions of $470,530,580$ and $620 \mathrm{~nm}$ alone maximizing the total luminous flux under $R_{i} \geqq 90$ at given correlated color temperatures.

The spectral power of $470 \mathrm{~nm}$, the shortest of the four wavelengths shown, increases with increasing CCT, but that of $620 \mathrm{~nm}$, the longest, decreases contrarily. The spectral powers of 530 and $580 \mathrm{~nm}$ remain nearly unchanged as CCT rises.

Figure 11 shows typical examples of SPD's. Figure 12 shows the characteristics of $R_{a}$ and $R_{i}$ $(i=9-15)$ against CCT. 

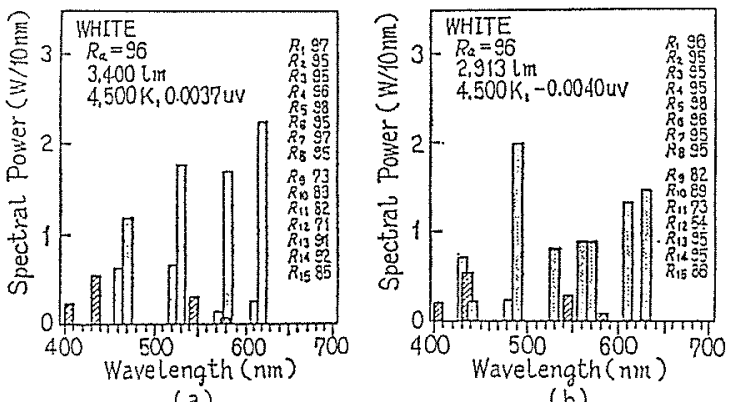

(a)

Fig. 11 Spectral power distributions of $470,530,580$ and $620 \mathrm{~nm}$ alone maximizing the total luminous flux at given chromaticity (hatched bars for the mercury spectral line).

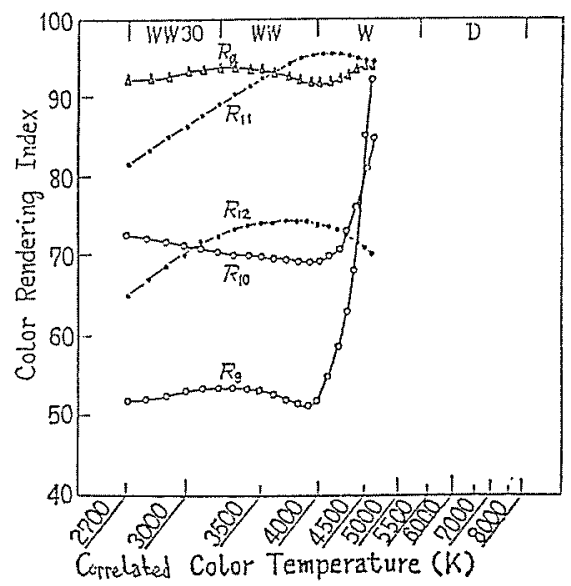

(a)

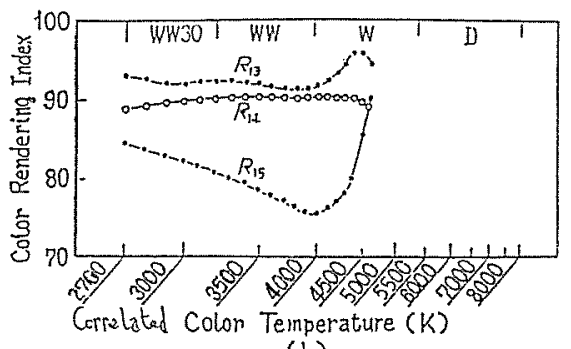

(b)

Fig. 12 Spectral power distributions of $470,530,580$ and $620 \mathrm{~nm}$ alone maximizing the total luminous flux under $R_{i} \geqq 90$ ( $i=1-8)$ at given correlated color temperatures.

The indices $R_{9}, R_{10}$ and $R_{15}$ markedly increase in the $W$ range where the total luminous flux decreases sharply. Figure 10 suggests that these merked increases are due to a slight decreases in the spectral power of $580 \mathrm{~nm}$ in the $W$ range.

The SPD in Fig. 6(a) has weak spectral powers at 480 and $520 \mathrm{~nm}$ in comparison with the one in Fig. 11(a). This difference alone causes such dif-

ferences in total luminous flux, $R_{a}$ and $R_{i}$ as shown in the figures. Also, the SPD in Fig. 11(b) composes of half as eight monochromatic components as the SPD in Fig. 5(a) and the former is lower than the latter in total luminous flux and $R_{a}$ by $1 \%$ and 2 respectively, but the former shows improvements over the latter in $R_{5}-R_{15}$ by $12,-2,12,2,-2$, -3 and 1 respectively.

The SPD's thus chosen by the process of optimization represent distributions of spectral powers of monochromatic components which are most effective in improving the total luminous flux and slight changes in each spectral power cause large change in the total luminous flux and CRI.

\section{Conclusion}

The visible wavelength range $400 \sim 700 \mathrm{~nm}$ of a $40 \mathrm{~W}$ fluorescent lamp was divided into 31 monochromatic components, each $10 \mathrm{~nm}$ in wavelength band width, and the optimum SPD was analyzed by applying LP to simultaneous equations consisting of the objective function maximizing the total Iuminous flux and the constraint equations on the radiant flux, chromaticity coordinates and special CRI's $R_{i}(i=1-8)$. The following conclusions were obtained.

(1) In conversion of ultraviolet exitation into spectral powers of the visible wavelength range by means of fluorescent materials, the highest luminous efficacy with satisfaction of given chromaticity and CRI can be produced by minimizing the number of monochromatic components and concentrating spectral powers to the chosen specific monochromatic components.

(2) Four monochromatic components, 470, 530, 580 and $620 \mathrm{~nm}$, are of primary importance and two, 460 and $520 \mathrm{~nm}$, are of secondary importance for the SPD's of fluorescent lamps of high color rendering properties which give the highest luminous efficacy and make all of the special CRI's of $R_{1}$ to $R_{g} 90$ or 95 or more.

Further improvements in the luminous efficacy and color rendering properties of fluorescent lamps can be expected in the future if fluorescent materials which show high quantum efficiency in a sufficiently narrow spectral band having a radiation peak at the above-mentioned specific monochromatic components are made available.

\section{References}

(1) Ivey, H. F.: J. Opt. Soc., 53 (1963) 1185.

(2) Koedam, M., Opstelten, J. J.: Lighting Res. Tech., 3 (1971) 205.

(3) Walter, W.: Illum. Engng., 7 (1978) 66.

(4) Fuchida, T., Akiyama, J. and Sugiyama, H.: J. Illum. Engng. Inst. Jpn., 63 (1979) 247.

(5) American National Standards Institute: "Specifications of the chromaticity of fluorescent lamps".

(6) Japanese Industrial Standards: "Chromaticity Classification of Fluorescent Lamps" JIS Z9112-1976, Jpn. Std. Assoc.

Received 30 Aug. 1984. 